Avaliação da Concentração de Mercúrio em Amostras de Biodiesel por Geração Fotoquímica de Vapor

\author{
Lisboa, M. T.; Ribeiro, A. S.; Vieira, M. A*
}

Rev. Virtual Quim., 2018, 10 (3), 609-624. Data de publicação na Web: 28 de junho de 2018

http://rvq.sbq.org.br

\title{
Evaluation of Mercury Concentration in Biodiesel Samples by Photochemical Vapor Generation
}

\begin{abstract}
In this paper, the photochemical vapor generation coupled with atomic absorption spectrometry was used to assess the concentration of $\mathrm{Hg}$ in biodiesel samples. The main parameters to assess the best conditions for the photochemical vapor generation of $\mathrm{Hg}$ were investigated (sample mass, formic acid concentration, irradiation time and gas flow rate). Volatile species of $\mathrm{Hg}$ were generated in biodiesel samples prepared in $20 \%(\mathrm{v} / \mathrm{v})$ formic acid and $76 \%(\mathrm{v} / \mathrm{v})$ ethanol. Analytical curves were generated using inorganic $\left(\mathrm{Hg}^{2+}\right)$ and organic standards $\left(\mathrm{CH}_{3} \mathrm{Hg}^{+}\right.$e $\left._{2} \mathrm{H}_{5} \mathrm{Hg}^{+}\right)$in ethanol and showed correlation coefficients higher than 0.99 . The limits of detection and quantification, in $\mu \mathrm{g} \mathrm{L}^{-1}$, were 0.01 and 0.05 , respectively. Due to the lack of biodiesel samples with a certified concentration of $\mathrm{Hg}$, the accuracy was evaluated by assessing the recoveries of species of $\mathrm{Hg}$ added to the samples, obtaining values ranged from 101.0 to $111.9 \%$ of recoveries. The relative standard deviation values were lower than $0.1 \%$, indicating an excellent precision of the method. No $\mathrm{Hg}$ was detected in the biodiesel samples, considering the obtained limit of detection. The proposed method is sensitive and the sample pre-treatment is simple and practical.
\end{abstract}

Keywords: Mercury; Biodiesel; Photochemical vapor generation; AAS.

\section{Resumo}

Neste trabalho, foi utilizada a geração fotoquímica de vapor acoplada à espectrometria de absorção atômica para avaliar a concentração de $\mathrm{Hg}$ em amostras de biodiesel. Os principais parâmetros para avaliar as melhores condições para a geração fotoquímica de vapor de $\mathrm{Hg}$ foram investigados (massa da amostra, concentração de ácido fórmico, tempo de irradiação e vazão do gás). As espécies voláteis de $\mathrm{Hg}$ foram geradas em amostras de biodiesel preparadas em $20 \%$ (v/v) ácido fórmico e $76 \%$ (v/v) etanol. As curvas analíticas foram preparadas usando padrões inorgânicos $\left(\mathrm{Hg}^{2+}\right)$ e orgânicos $\left(\mathrm{CH}_{3} \mathrm{Hg}^{+}\right.$e $\left.\mathrm{C}_{2} \mathrm{H}_{5} \mathrm{Hg}^{+}\right)$ em etanol e mostraram coeficientes de correlação maiores que 0,99. Os limites de detecção e quantificação, em $\mu \mathrm{g} \mathrm{L} \mathrm{L}^{-1}$, foram 0,01 e 0,05 , respectivamente. Devido à falta de amostras de biodiesel com uma concentração certificada de $\mathrm{Hg}$, a exatidão foi avaliada através das recuperações de espécies de $\mathrm{Hg}$ adicionadas às amostras, obtendo-se valores de recuperação entre 101,0 a 111,9\%, sendo estes valores aceitáveis para as condições empregadas para as análises. Os valores do desvio padrão relativo foram inferiores a $0,1 \%$, indicando uma excelente precisão do método. Não foi detectado $\mathrm{Hg}$ nas amostras de biodiesel, considerando o limite de detecção obtido. O método proposto mostrou boa sensibilidade e o preparo da amostra é simples e prático.

Palavras-chave: Mercúrio; Biodiesel; Geração fotoquímica de vapor; AAS.

\footnotetext{
* Universidade Federal de Pelotas, Programa de Pós-Graduação em Química, Laboratório de Metrologia Química (LabMeQui), CEP 96160-000, Capão do Leão-RS, Brasil.

$M$ marianavieira@pq.cnpq.br DOI: $\underline{10.21577 / 1984-6835.20180045}$
}

Rev. Virtual Quim. |Vol 10| |No. 3| |609-624| 


\title{
Avaliação da Concentração de Mercúrio em Amostras de Biodiesel por Geração Fotoquímica de Vapor
}

\author{
Meibel T. Lisboa, Anderson S. Ribeiro, Mariana A. Vieira*
}

Universidade Federal de Pelotas, Programa de Pós-Graduação em Química, Laboratório de Metrologia Química (LabMeQui), CEP 96160-000, Capão do Leão-RS, Brasil.

* marianavieira@pq.cnpq.br

Recebido em 14 de janeiro de 2018. Aceito para publicação em 25 de junho de 2018

\section{Introdução}

\section{Parte Experimental}

\subsection{Instrumentação}

2.1. Reagentes e materiais

2.3. Tratamento da amostra

2.4. Estudos do sistema PVG- CV AAS

2.5. Parâmetros de mérito

\section{Resultados e Discussão}

3.1. Efeito da concentração de ácido fórmico

3.1. Efeito da massa da amostra

3.3. Efeito da vazão do gás carreador

3.4. Efeito do tempo de irradiação da amostra

3.5. Parâmetros de mérito

\section{Conclusão}

\section{Introdução}

A alta demanda de energia no mundo industrializado e no setor doméstico, bem como os problemas de poluição causados devido ao vasto uso dos combustíveis de origem fóssil, têm resultado em uma crescente necessidade de se utilizar fontes de energias renováveis de menor impacto ambiental. Neste contexto, os biocombustíveis que são derivados de biomassa renovável podem substituir, parcial ou totalmente, os combustíveis derivados de petróleo e gás natural em motores à combustão ou em outro tipo de geração de energia. ${ }^{1}$

O biodiesel apresenta vantagens sobre 0 diesel de petróleo. É um combustível renovável obtido a partir de um processo químico denominado transesterificação, onde os triacilgliceróis presentes nos óleos e gordura animal reagem com álcoois de cadeia curta (metanol ou etanol), gerando dois produtos: o éster e o glicerol. ${ }^{2}$ Uma importante questão na utilização de um 
combustível é a avaliação qualitativa e quantitativa de elementos em sua composição. Estes podem ser incorporados durante a extração de óleo ou gordura e durante a produção, lavagem, refino, transporte e armazenamento do biodiesel. ${ }^{3}$ No Brasil, a Agência Nacional do Petróleo, Gás Natural e Biocombustíveis (ANP), na Resolução ANP no 45 de 2014 estabelece os limites máximos de concentração para alguns elementos no biodiesel que é comercializado em todo território nacional: $\mathrm{Na}+\mathrm{K}(5 \mathrm{mg} \mathrm{kg}$ $\left.{ }^{1}\right) ; \mathrm{Ca}+\mathrm{Mg}\left(5 \mathrm{mg} \mathrm{kg}^{-1}\right) ; \mathrm{S}\left(10 \mathrm{mg} \mathrm{kg}^{-1}\right)$ e P (10 $\left.\mathrm{mg} \mathrm{kg}{ }^{-1}\right) .{ }^{4}$ No entanto, as especificações para o biodiesel não contemplam a determinação e os níveis máximos permitidos para $\mathrm{Hg}$, As e Si. Estes elementos podem estar presentes nas fontes primárias de obtenção do biodiesel, tornando-o vulnerável a contaminações em seu processo de obtenção e danoso ao ecossistema ${ }^{5,6}$ Assim, é de suma importância quantificar $\mathrm{Hg}$ e outros metais que possam interferir no propósito da utilização do biodiesel como uma fonte "verde" de combustível.

Segundo Almeida e colaboradores ${ }^{7}$, a rota provável para a contaminação do biodiesel e seu coproduto por $\mathrm{Hg}$ pode ocorrer por diversos fatores, como a incorporação do metal durante o crescimento da planta, uso intensivo de fertilizantes, contaminação do solo por esgotos e até mesmo emissões de forma natural ou por ações antropogênicas. $\mathrm{O} \mathrm{Hg}$ é liberado pelo processo de combustão dos combustíveis, tornando-o uma importante fonte de poluição atmosférica. É também considerado um elemento altamente tóxico, mesmo em baixas concentrações, devido ao seu poder acumulativo no meio ambiente e sua toxicidade depende da forma química em que ele se encontra: $\mathrm{C}_{2} \mathrm{H}_{5} \mathrm{Hg}^{+}>\mathrm{CH}_{3} \mathrm{Hg}^{+}>\mathrm{Hg}^{0}>$ $\mathrm{Hg}^{2+8}$.

Diversas técnicas para a determinação de $\mathrm{Hg}$ podem ser empregadas, tais como a espectrometria de fluorescência atômica com geração de vapor frio (CV AFS), a espectrometria de emissão óptica com plasma indutivamente acoplado (ICP OES); a espectrometria de massas com plasma indutivamente acoplado (ICP-MS) e a espectrometria de absorção atômica com vapor frio (CV AAS), sendo esta última a mais popular. ${ }^{9} \mathrm{Na}$ CV AAS, as espécies de $\mathrm{Hg}$ são normalmente mineralizadas a $\mathrm{Hg}^{2+}$ por meio de um ataque ácido e, em seguida, reduzidas para a sua forma elementar $\left(\mathrm{Hg}^{0}\right)$ por meio de reação com agentes redutores como o $\mathrm{SnCl}_{2}$ ou o $\mathrm{NaBH}_{4}{ }^{8}$ A fim de reduzir ou eliminar a utilização de agentes redutores e contribuir com a química verde, a geração fotoquímica de vapor acoplada à espectrometria de absorção atômica com vapor frio (PVG-CV AAS) é uma técnica emergente que vem sendo utilizada com êxito na determinação de $\mathrm{Hg}$ e outros elementos em diferentes tipos de amostras, como material bológico ${ }^{10,11}$, álcool combustível $^{11,12}$, glicerina oriunda do processo de biodiesel ${ }^{13}$, nafta e petróleo ${ }^{14}$, entre outras.

A geração fotoquímica de vapor (PVG, do inglês photochemical vapor generation) baseia-se na exposição da amostra em presença de ácidos orgânicos de baixo peso molecular a um campo de radiação ultravioleta, resultando na formação de produtos intermediários. Nesse sistema, a amostra é misturada com um fluxo de gás inerte e introduzida em um separador gáslíquido, onde as espécies voláteis resultantes são direcionadas até a cela de absorção para posterior detecção. ${ }^{15,16}$ A eficiência do sistema PVG em promover a geração de vapor de $\mathrm{Hg}^{0}$ independe da espécie de $\mathrm{Hg}$ presente na solução (orgânico ou inorgânico), porque os mesmos são degradados pela clivagem das ligações $\mathrm{Hg}-\mathrm{C}$, seguido por redução de $\mathrm{Hg}^{2+}$ para $\mathrm{Hg}^{0}$, como demonstrado em trabalhos anteriores do nosso grupo de pesquisa. ${ }^{17,18}$

Desta forma, este estudo teve como objetivo, avaliar a viabilidade de quantificar $\mathrm{Hg}$ em amostras de biodiesel de soja (B100) utilizando a geração fotoquímica de vapor como meio de introdução das amostras na técnica de CV AAS. 


\section{Parte Experimental}

\subsection{Instrumentação}

Para as medidas de sinal de absorvância do $\mathrm{Hg}$, foi utilizado um espectrômetro de absorção atômica modelo AA-6300 (Shimadzu, Japão) equipado com uma lâmpada de cátodo oco de $\mathrm{Hg}$ (Hamamatsu photonics K.K, Japão) e corretor de fundo com lâmpada de arco de deutério. Foi selecionado o comprimento de onda de $253,7 \mathrm{~nm}$, fenda espectral de $0,7 \mathrm{~nm}$ e corrente da lâmpada de 4,0 mA.
Um atomizador de tubo de quartzo (QTA) foi posicionado no caminho óptico do espectrômetro e mantido a temperatura ambiente. $O$ reator fotoquímico foi construído com uma lâmpada de UV de baixa pressão de vapor de $\mathrm{Hg}(254 \mathrm{~nm}, 15 \mathrm{~W}$, Cole Parmer, EUA) e o percurso analítico pelo qual as soluções percorreram foi construído com tubos de quartzo envoltos na lâmpada de UV, com um volume interno de aproximadamente $26,0 \mathrm{~mL}$, formando assim uma bobina de reação. A Figura 1 mostra o reator fotoquímico utilizado neste trabalho. Ressaltamos que o sistema é todo envolto com papel alumínio e são utilizados óculos de proteção para evitar exposição à radiação pelo operador quando a lâmpada está ligada.

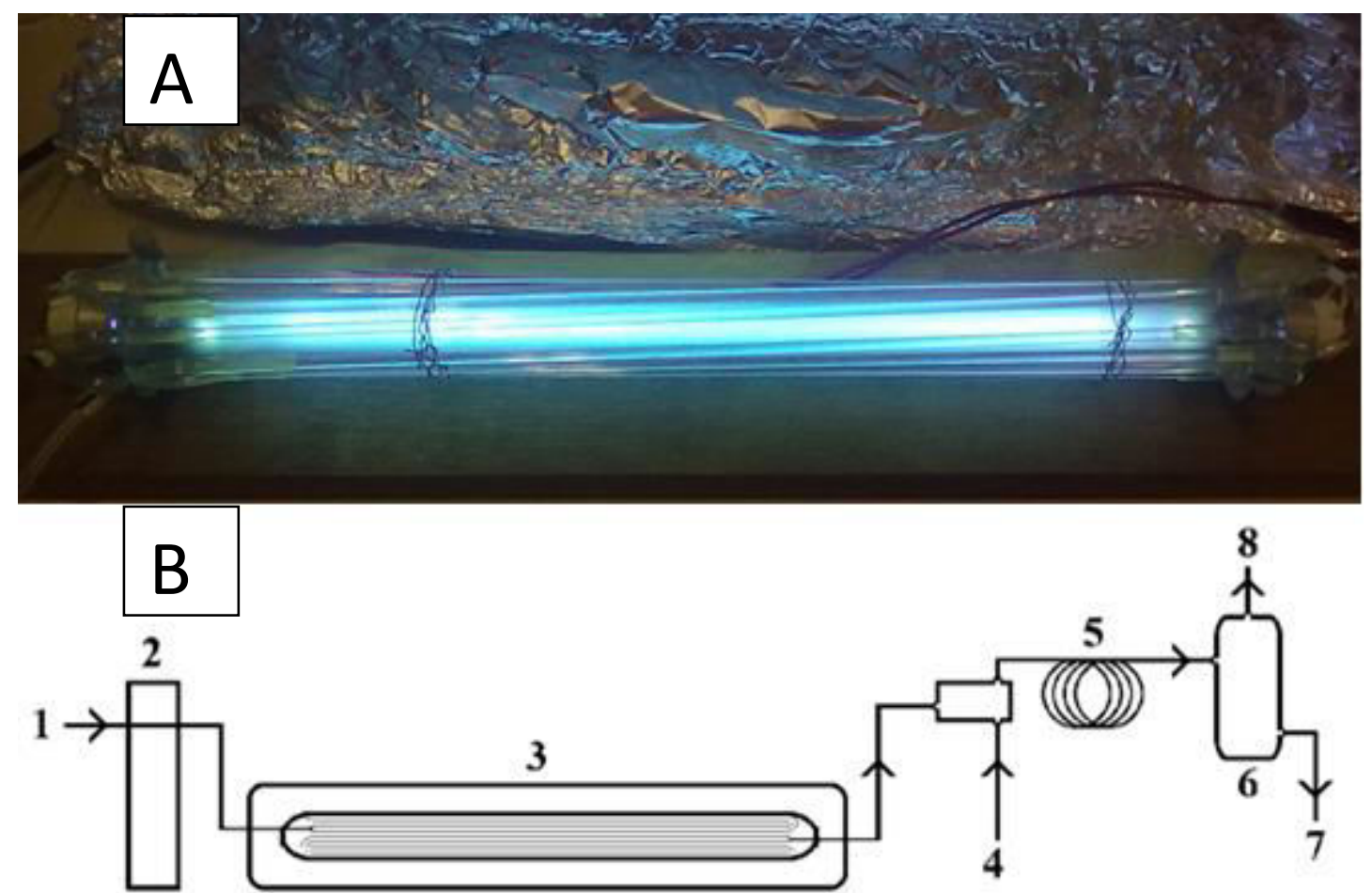

Figura 1. A) Reator fotoquímico. B) Sistema de geração fotoquímica:1: amostra, 2: bomba peristáltica, 3: reator fotoquímico, 4: entrada de gás $\mathrm{Ar}, 5$ : bobina de reação, 6: separador gáslíquido, 7: descarte, 8: tubo $T$ de quartzo

As soluções foram transportadas pelo reator fotoquímico através de uma bomba peristáltica Ismatec modelo CP-78017-35 (Cole Parmer, Vernon Hills, EUA) com uma vazão de $7,0 \mathrm{~mL} \mathrm{~min}{ }^{-1}$ que equivale a uma velocidade de rotação da bomba peristáltica de $70 \mathrm{rpm}$ e tempo de irradiação da amostra de 3,7 minutos. A vazão do gás de arraste $\mathrm{Ar}$ foi controlada por meio de um fluxômetro modelo 03217-10 de 150 mm (Cole Parmer, 
EUA), o qual foi ajustado em uma vazão de $62,6 \mathrm{~mL} \mathrm{~min}{ }^{-1}$. As amostras irradiadas foram conduzidas a um separador gás-líquido e as espécies voláteis formadas foram transportadas diretamente para o atomizador com o auxílio do gás argônio com uma pureza de 99,996\% (Linde, Barueri, SP, Brasil).

\subsection{Reagentes e Materiais}

Todos os reagentes utilizados foram de grau analítico e todas as soluções foram preparadas utilizando água deionizada. Uma solução estoque de $1.000 \mathrm{mg} \mathrm{L}^{-1}$ de $\mathrm{Hg}^{2+}$ foi preparada pela diluição de uma ampola Titrisol (Merck, Darmstadt, Alemanha) em $0,5 \%(\mathrm{v} / \mathrm{v})$ de $\mathrm{HNO}_{3}$. Soluções das espécies organometálicas de $\mathrm{Hg}$ foram obtidas de forma similar, onde $1000 \mathrm{mg} \mathrm{L}^{-1}$ de $\mathrm{CH}_{3} \mathrm{Hg}^{+}$e $400 \mathrm{mg} \mathrm{L}^{-1}$ de $\mathrm{C}_{2} \mathrm{H}_{5} \mathrm{Hg}^{+}$foram preparadas pela dissolução do sal de cloreto de metilmercúrio (Fluka Analytical, Steinheim, Alemanha) e cloreto de etilmercúrio (Analytical Supelco, Bellefonte, EUA), respectivamente em metanol.

Também foram utilizados reagentes como álcool etílico $\left(\mathrm{CH}_{3} \mathrm{CH}_{2} \mathrm{OH}\right)$ 99,5\% (v/v) (Synth) que foi destilado em um sistema de destilação convencional de vidro e ácido fórmico $(\mathrm{HCOOH}) 85 \%(\mathrm{v} / \mathrm{v})$ (Synth) que foi destilado em um destilador de quartzo MA075 (Marconi, Piracicaba, SP, Brasil). Esse procedimento foi necessário para assegurar a remoção de impurezas dos reagentes que podem afetar a eficiência da geração de vapor no sistema fotoquímico. As amostras de biodiesel foram pesadas em uma balança analítica Ohaus Adventurer modelo AR 2140 (Pine Brook, NJ, EUA) com resolução de 0,1 mg e tara máxima de $210 \mathrm{~g}$.

\subsection{Tratamento da amostra}

Para os estudos, utilizaram-se três amostras de biodiesel de soja (B100) denominadas de Biodiesel 1, Biodiesel 2 e Biodiesel 3. Todas as amostras foram gentilmente fornecidas por diferentes plantas de produção de biodiesel localizadas no estado do Rio Grande do Sul.

Para as análises, as mesmas foram preparadas da seguinte maneira: aproximadamente $2,0 \mathrm{~g}$ de amostra foram pesadas diretamente em tubos de polipropileno (PP), onde foram adicionados $11,8 \mathrm{~mL}$ de $\mathrm{HCOOH} 85 \%$ (v/v) e o volume final de $50 \mathrm{~mL}$ foi completado com $\mathrm{CH}_{3} \mathrm{CH}_{2} \mathrm{OH}$, resultando em uma concentração final de $20 \%(\mathrm{v} / \mathrm{v})$ de $\mathrm{HCOOH}$ e $76,0 \%(\mathrm{v} / \mathrm{v})$ de $\mathrm{CH}_{3} \mathrm{CH}_{2} \mathrm{OH}$.

\subsection{Estudos do sistema PVG-CV AAS}

Para obtenção de uma maior eficiência do sistema de geração fotoquímica de vapor, os seguintes parâmetros foram estudados: concentração de $\mathrm{HCOOH}$ no intervalo de 5,0 a $30,0 \%(\mathrm{v} / \mathrm{v})$; massa de amostra $(0,25$ a 2,00 g); vazão do gás carreador $\operatorname{Ar}(62,6$ a 126,0 $\left.\mathrm{mL} \mathrm{min}^{-1}\right)$ e tempo de irradiação $(2,7$ a 8,7 min). Para estes estudos, foram utilizadas soluções padrão contendo $5,0 \mu \mathrm{g} \mathrm{L}^{-1}$ de $\mathrm{Hg}^{2+}$ sem e com a presença da amostra de biodiesel 1 em um volume final de solução de $50 \mathrm{~mL}$.

Para o estudo da vazão do gás carreador Ar, foi utilizado um fluxômetro. O tempo de irradiação foi calculado conforme as variações de velocidade de rotação da bomba peristáltica. Para cada velocidade, uma vazão de solução é obtida (Tabela 1) e o tempo de irradiação é então calculado levando em consideração o comprimento do tubo de quartzo envolto no reator fotoquímico, que possui um volume interno de $26 \mathrm{~mL}$. 
Tabela 1. Tempo de irradiação das soluções em função da velocidade da bomba peristáltica para o sistema PVG-CV AAS

\begin{tabular}{ccc}
\hline $\begin{array}{c}\text { Velocidade de rotação da } \\
\text { bomba peristáltica (rpm) }\end{array}$ & $\begin{array}{c}\text { Vazão da solução } \\
\left(\mathbf{m L ~ m ~}^{-\mathbf{1}}\right)\end{array}$ & $\begin{array}{c}\text { Tempo de irradiação } \\
(\mathbf{m i n})\end{array}$ \\
\hline 30 & 3,0 & 8,7 \\
40 & 4,0 & 6,5 \\
50 & 5,0 & 5,2 \\
60 & 6,0 & 4,3 \\
70 & 7,0 & 3,7 \\
80 & 8,0 & 3,2 \\
90 & 9,0 & 2,9 \\
\hline
\end{tabular}

\subsection{Parâmetros de Mérito}

Os parâmetros de mérito avaliados para a validação do método desenvolvido foram: limites de detecção e quantificação, linearidade, precisão e exatidão. Todos os cálculos empregados estão baseados nas orientações do guia de validação do INMETRO. ${ }^{19}$

Duas curvas analíticas foram construídas de modo a avaliar qual apresentaria a melhor sensibilidade. A primeira curva foi obtida com padrões de $\mathrm{Hg}^{2+}$ em meio de $20 \%(\mathrm{v} / \mathrm{v})$ de $\mathrm{HCOOH}$ e $76 \%$ (v/v) de $\mathrm{CH}_{3} \mathrm{CH}_{2} \mathrm{OH}$ (meio alcoólico) e a segunda curva foi obtida nas mesmas condições, mas na presença da matriz (biodiesel). A faixa linear de calibração utilizada em ambos os casos foi de até 10,0 $\mu \mathrm{g} \mathrm{L}^{-1}$ de $\mathrm{Hg}^{2+}$. Foram preparados padrões de calibração nas seguintes concentrações: 2,5; 5,0; 7,5 e $10 \mu \mathrm{g} \mathrm{L}^{-1} \mathrm{de} \mathrm{Hg}^{2+}$.

Também, foram construídas curvas analíticas com padrões de $\mathrm{Hg}^{2+}, \mathrm{CH}_{3} \mathrm{Hg}^{+}$e $\mathrm{C}_{2} \mathrm{H}_{5} \mathrm{Hg}^{+}$, em uma faixa linear de 2,5 a $10,0 \mu \mathrm{g}$ $\mathrm{L}^{-1}$ em meio de $20 \%(\mathrm{v} / \mathrm{v})$ de $\mathrm{HCOOH}$ e avolumadas a $50,00 \mathrm{~mL}$ com $\mathrm{CH}_{3} \mathrm{CH}_{2} \mathrm{OH}$.

Os limites de detecção (LD) e quantificação (LQ) foram calculados de acordo com as equações 1 e 2 , respectivamente: ${ }^{19}$

$$
\begin{aligned}
& L D=3(S / a) \\
& L Q=10(S / a)
\end{aligned}
$$

onde S é a estimativa de desvio-padrão de 10 leituras do branco analítico e "a" é a inclinação da curva analítica.

A linearidade foi avaliada pelo cálculo do coeficiente de correlação da curva analítica (R). Foram consideradas como satisfatórias as curvas com valores de $R$ maiores ou iguais a 0,99 . A precisão foi avaliada através da estimativa do desvio padrão relativo das leituras (DPR) e calculada pela Equação $3:{ }^{19}$

$$
\operatorname{DPR}(\%)=(\mathrm{S} / \mathrm{X}) \times 100
$$

onde $S$ é igual a estimativa de desvio-padrão das medidas, $X$ é igual a concentração média. Foram usados como valores de referência aceitáveis, os descritos pela $\mathrm{AOAC}^{20}$

Devido à inexistência de materiais de referência certificados para $\mathrm{Hg}$ em amostras de biodiesel, a exatidão foi avaliada através do teste de adição e recuperação do analito. Foram feitas adições nas amostras de biodiesel em três níveis de concentração, usando uma mistura contendo as três diferentes espécies de $\mathrm{Hg}$ nas concentrações totais de 3,0; 6,0 e 9,0 $\mu \mathrm{g} \mathrm{L}^{-1}$ (por exemplo, se 
a concentração total foi de $3,0 \mu \mathrm{g} \mathrm{L}^{-1}$, foi adicionado 1,0 $\mathrm{g} \mathrm{L} \mathrm{L}^{-1}$ de cada espécie). Os valores de recuperação foram calculados com auxílio da equação 4 :

$$
\operatorname{Rec}(\%)=\left(C / C_{A D I}\right) \times 100
$$

onde $C$ é a concentração média das recuperações obtidas para $n$ repetições e $C_{A D I}$ é a concentração adicionada. Considerou-se o intervalo de recuperação de 80 a $120 \%$ como suficiente para aceitação da exatidão do método empregado. ${ }^{19}$

\section{Resultados e Discussão}

De modo a avaliar a potencialidade da técnica de geração fotoquímica de vapor acoplada a AAS para a avaliação da concentração de $\mathrm{Hg}$ em amostras de biodiesel, alguns parâmetros essenciais para obtenção de uma maior eficiência do sistema foram investigados. Todos os estudos foram conduzidos utilizando soluções padrão de $\mathrm{Hg}^{2+}$ como descrito na parte experimental (Secção 2.3.) e o sistema com o reator fotoquímico mostrado na Figura 1.

\subsection{Efeito da concentração de ácido fórmico}

Os ácidos orgânicos de baixo peso molecular são comumente utilizados em estudos que envolvem a PVG através da exposição à radiação UV, devido a uma elevada eficiência em produzir radicais reativos que se combinam com alguns elementos, como $\mathrm{Hg}$, As, Se, entre outros, formando espécies voláteis. ${ }^{21,22}$ Dentre estes ácidos, o ácido fórmico apresenta-se como um excelente precursor para a geração de espécies voláteis, principalmente de $\mathrm{Hg}$, como relatado em estudos anteriores para diferentes tipos de amostras. ${ }^{11,17}$ Sendo assim, o efeito da concentração de $\mathrm{HCOOH}$ no sinal de absorvância do $\mathrm{Hg}$ foi avaliado e a Figura 2 apresenta os resultados obtidos.

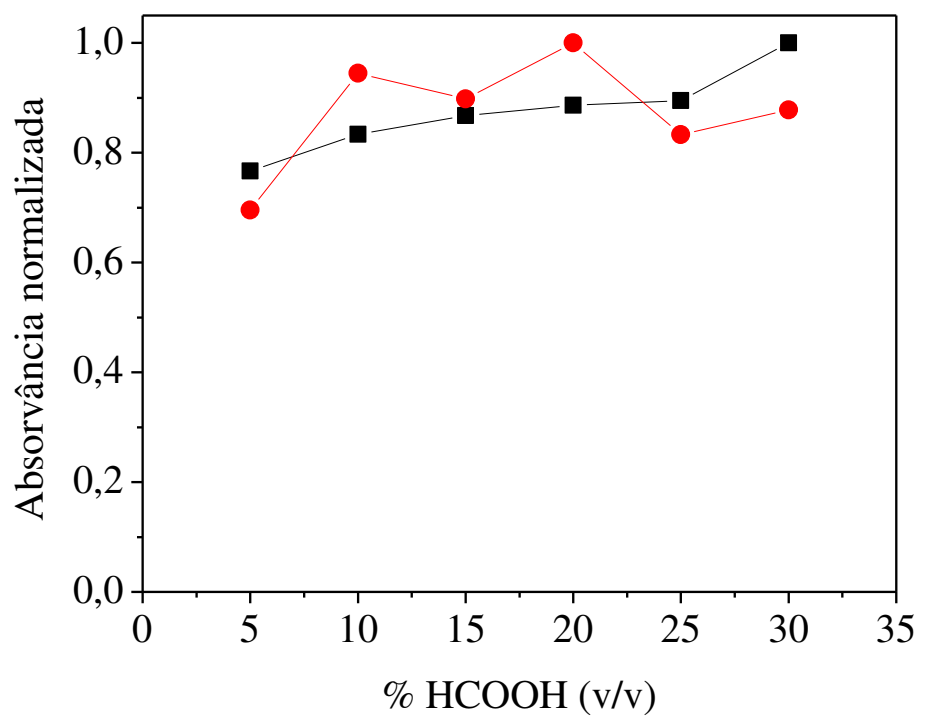

Figura 2. Efeito da concentração de $\mathrm{HCOOH}$ no sinal de absorvância de Hg após PVG-CV AAS em (--) padrão aquoso com $5 \mu \mathrm{g} \mathrm{L}^{-1} \mathrm{de} \mathrm{Hg}^{2+}+1,0 \mathrm{~g}$ de biodiesel $1 \mathrm{e}(-\bullet-)$ padrão aquoso com

$5 \mu \mathrm{g} \mathrm{L}^{-1}$ de $\mathrm{Hg}^{2+}$. Tempo de irradiação: 3,7 minutos; Vazão do gás carreador: 95,4 $\mathrm{mL} \mathrm{min}^{-1}$ 
De acordo com os resultados mostrados na Figura 2, foi observado um aumento no sinal de absorvância de $\mathrm{Hg}$ na solução contendo a amostra. Para o padrão aquoso, o sinal foi maior para a concentração de $20 \%$ (v/v) de $\mathrm{HCOOH}$ e após esta, houve uma queda. Desta forma, ficou evidente que em meio da amostra, há um aumento significativo da concentração de radicais formados após a exposição à radiação UV, aumentando desta forma a sensibilidade. Assim, foi fixada a concentração de $20 \%$ (v/v) de $\mathrm{HCOOH}$ para os estudos seguintes.

\subsection{Efeito da massa da amostra}

Por se tratar de uma matriz que normalmente não apresenta quantidades detectáveis de $\mathrm{Hg}$, foi realizado um estudo para avaliar a quantidade de amostra a ser utilizada, de modo a obter uma maior sensibilidade durante o processo de redução fotoquímica e também um melhor limite de detecção. A Figura 3 apresenta os resultados obtidos para este estudo.

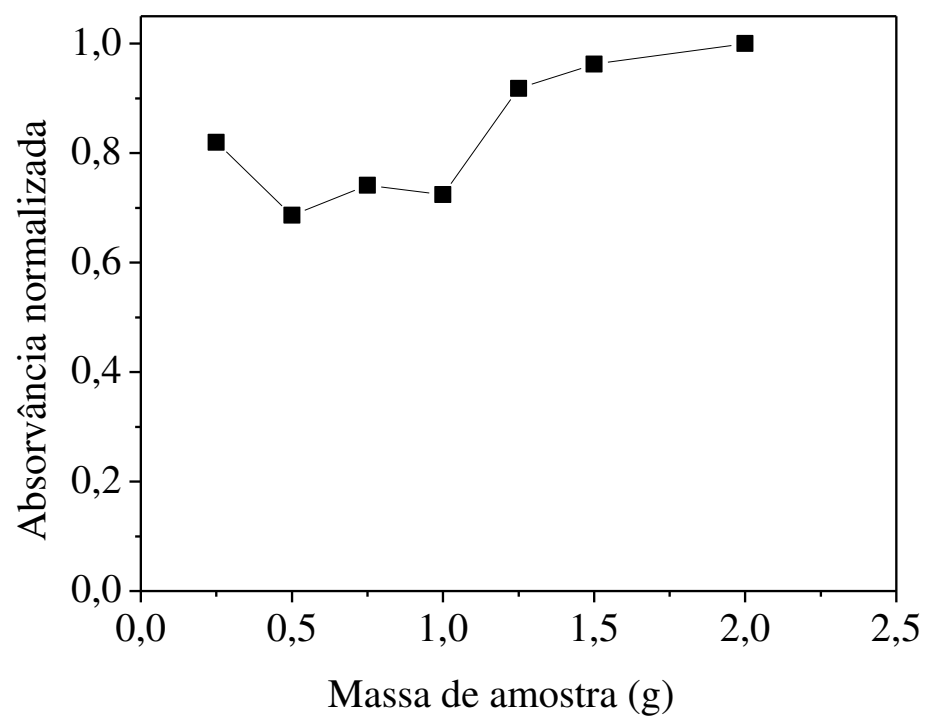

Figura 3. Efeito da massa da amostra no sinal de absorvância de Hg após PVG-CV AAS. Soluções contendo $5 \mathrm{\mu g} \mathrm{L}^{-1}$ de $\mathrm{Hg}^{2+}$ em $20 \%$ (v/v) de $\mathrm{HCOOH}$ e $76 \%$ (v/v) de $\mathrm{CH}_{3} \mathrm{CH}_{2} \mathrm{OH}$. Tempo de irradiação: 3,7 minutos; Vazão do gás carreador: 95,4 $\mathrm{mL} \mathrm{min}^{-1}$

De acordo com os resultados apresentados na Figura 3, foi observado que os sinais de absorvância para $\mathrm{Hg}$ foram melhores para massas de amostras acima de $1,25 \mathrm{~g}$, e o sinal mais intenso foi observado em $2,0 \mathrm{~g}$ de amostra, sendo esta a escolhida para os estudos subsequentes.

\subsection{Efeito da vazão do gás carreador}

A vazão do gás carreador é importante durante o processo de geração fotoquímica de vapor, uma vez que este tem a função de transportar as espécies voláteis geradas até a cela de absorção. Desta forma, um estudo para avaliar a melhor vazão do gás $\mathrm{Ar}$ foi realizado e os resultados obtidos são apresentados na Figura 4. 


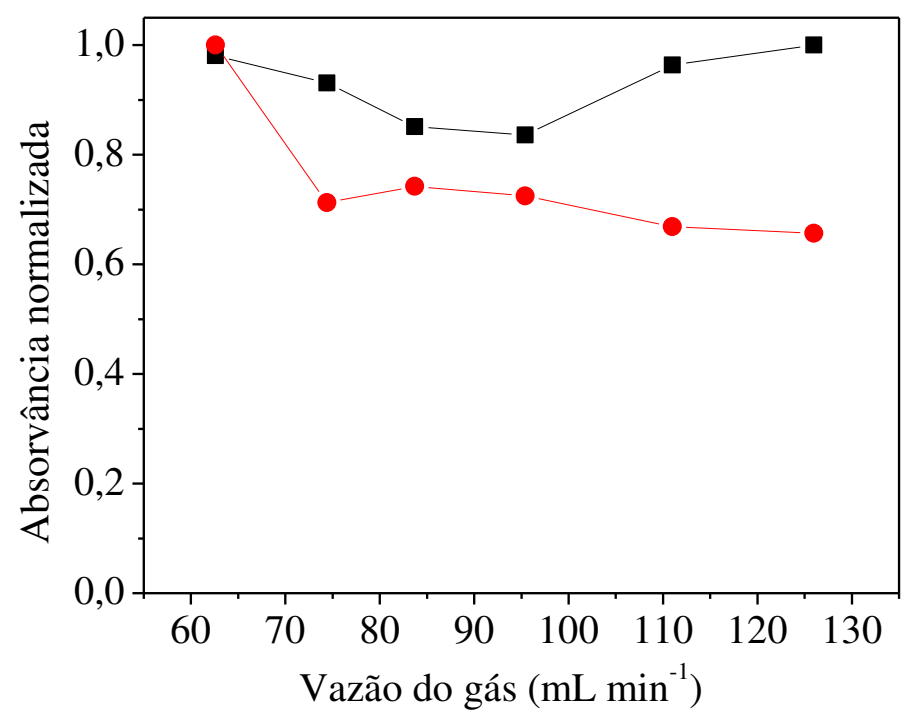

Figura 4. Efeito da vazão do gás carreador no sinal de absorvância de Hg após PVG-CV AAS. (--) padrão aquoso com $5 \mu \mathrm{g} \mathrm{L}^{-1} \mathrm{de} \mathrm{Hg}^{2+}+2,0 \mathrm{~g}$ de biodiesel 1 ; (-๑-) padrão aquoso com $5 \mu \mathrm{g} \mathrm{L}$ ${ }^{1}$ de $\mathrm{Hg}^{2+}$. Soluções preparadas em $20 \%$ (v/v) de $\mathrm{HCOOH}$ e $76 \%(\mathrm{v} / \mathrm{v})$ de $\mathrm{CH}_{3} \mathrm{CH}_{2} \mathrm{OH}$; Tempo de irradiação: 3,7 minutos

De acordo com os resultados apresentados na Figura 4, observou-se um maior sinal nos dois meios avaliados quando a menor vazão do gás foi utilizada $(62,6 \mathrm{~mL}$ $\left.\mathrm{min}^{-1}\right)$. À medida que a vazão do gás aumenta, o sinal de absorvância tende a diminuir em função do efeito de diluição da nuvem atômica que chega até a cela de absorção. Sendo assim, a vazão de $62,6 \mathrm{~mL}$ $\mathrm{min}^{-1}$ foi escolhida como a condição adequada para a obtenção de um bom sinal analítico de $\mathrm{Hg}$ nos dois meios estudados, sem prejudicar a sensibilidade das medidas e também a frequência analítica, que foi em torno de 6 amostras por hora. Ressalta-se que as amostras de biodiesel não receberam nenhum tratamento prévio, mas sim uma simples diluição. Desta forma, a baixa frequência analítica é compensada pela simplicidade do método com um número bastante reduzido de etapas. Além disso, o $\mathrm{Hg}$ é extremamente volátil e quando se faz necessário a mineralização da amostra, a etapa de preparo geralmente é onerosa e pode se tornar um grande desafio, quando não conduzido em equipamentos apropriados e por profissionais altamente treinados podendo inviabilizar as análises.

\subsection{Efeito do tempo de irradiação da amostra}

O tempo de exposição das amostras à radiação UV no percurso analítico do reator fotoquímico é considerado um parâmetro fundamental a ser avaliado na PVG-AAS, já que a quantidade de radiação UV recebida irá determinar a extensão da formação dos radicais produzidos, que está diretamente relacionada à eficiência de redução do $\mathrm{Hg}^{2+}$. Sendo assim, o efeito do tempo de irradiação foi estudado e os resultados obtidos são apresentados na Figura 5.

A Figura 5 mostra o estudo realizado para avaliar a influência do tempo de irradiação da amostra no sinal de absorvância do $\mathrm{Hg}$, através da variação velocidade de rotação da bomba peristáltica, na faixa de 30 a 90 rpm, o que corresponde a um tempo de irradiação de 8,7 a 2,9 min e a uma vazão de amostra de 3,0 a $9,0 \mathrm{~mL} \mathrm{~min}^{-1}$, respectivamente. 


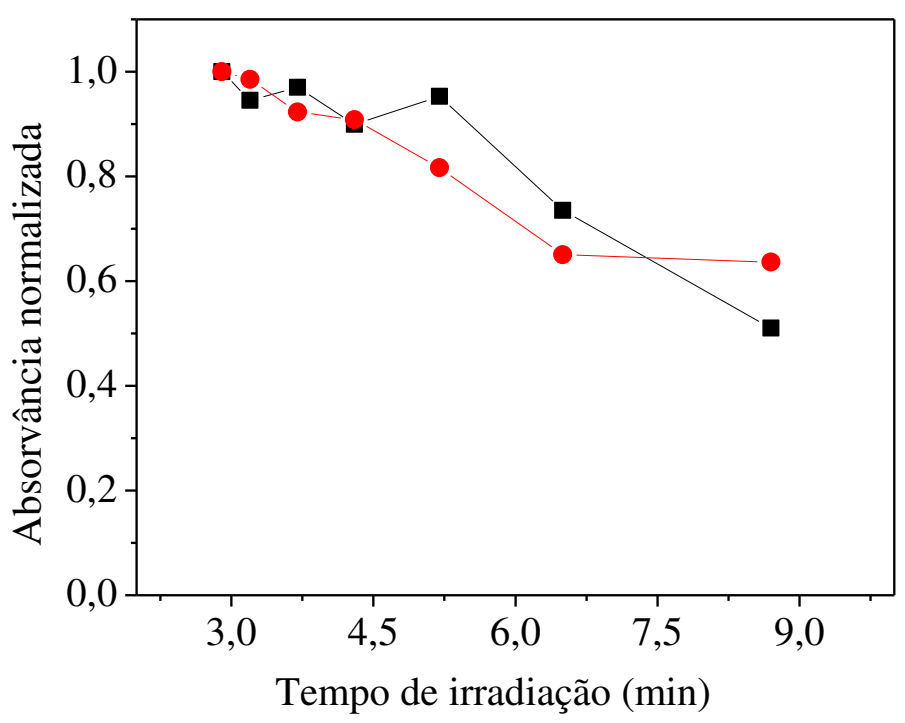

Figura 5. Efeito do tempo de irradiação da solução no sinal de absorvância de Hg por PVG-CV

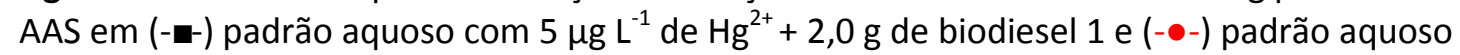
com $5 \mu \mathrm{g} \mathrm{L}^{-1}$ de $\mathrm{Hg}^{2+}$. Soluções preparadas em $20 \%$ (v/v) de $\mathrm{HCOOH}$ e $76 \%(\mathrm{v} / \mathrm{v})$ de $\mathrm{CH}_{3} \mathrm{CH}_{2} \mathrm{OH}$; Vazão do gás carreador: $62,6 \mathrm{~mL} \mathrm{~min}^{-1}$

Os resultados apresentados na Figura 5 mostraram que um tempo de irradiação de 2,9 min seria o ideal, o qual representa uma vazão de amostra de $9,0 \mathrm{~mL} \mathrm{~min}^{-1}$ e uma velocidade de rotação da bomba peristáltica de $90 \mathrm{rpm}$. No entanto, também foi observado que quando se utiliza altas velocidades de rotação, os tubos acabam sendo danificados e comprometem a eficiência do sistema. Por conta disso, para que não houvesse perda de sensibilidade em nenhum dos dois meios avaliados, foi adotado um tempo de irradiação de 3,7 min, que corresponde a uma vazão de $7,0 \mathrm{~mL} \mathrm{~min}$ ${ }^{1}$ e uma velocidade de rotação da bomba de $70 \mathrm{rpm}$.

Este estudo demonstra que com o aumento da velocidade da bomba peristáltica, embora diminua o tempo de irradiação, houve um maior processamento da amostra no sistema fotoquímico em um menor tempo, fornecendo uma nuvem atômica mais concentrada, refletindo no aumento do sinal e consequentemente, na maior sensibilidade do sistema PVG-CV AAS para a redução de $\mathrm{Hg}$.

Este fato mostra que o reator fotoquímico utilizado apresenta uma eficiência elevada para geração dos radicais.

\subsection{Parâmetros de mérito}

Depois de estabelecidas as melhores condições de trabalho do sistema de geração fotoquímica de vapor, foram construídas duas curvas analíticas de modo a avaliar a capacidade das mesmas em fornecer resultados exatos visando a quantificação de Hg nas amostras de biodiesel. Os parâmetros de mérito obtidos são apresentados na Tabela 2. 
Tabela 2. Parâmetros de mérito para as curvas analíticas obtidas em diferentes meios

\begin{tabular}{ccccc}
\hline & $\mathbf{a}\left({\mathrm{L} ~ \mu \mathrm{g}^{-1}}^{-1}\right.$ & $\mathbf{R}$ & $\mathbf{L D}\left(\mu \mathrm{g} \mathrm{L}^{-1}\right)^{*}$ & $\mathbf{L Q}\left(\mu \mathrm{g} \mathrm{L}^{-1}\right)^{*}$ \\
\hline Meio alcoólico & 0,0119 & 0,9975 & 0,01 & 0,05 \\
Meio de biodiesel & 0,0095 & 0,9993 & 0,06 & 0,21
\end{tabular}

*LD e LQ instrumental

a: coeficiente angular

R: coeficiente de correlação da curva analítica

Os resultados demonstraram uma boa linearidade das curvas, com coeficientes de correlação linear (R) maiores que 0,99 e os valores das inclinações das curvas demonstraram que a curva em meio alcoólico apresentou uma maior sensibilidade quando comparada a curva na presença da amostra, com uma diferença de $20 \%$ entre os valores obtidos, sendo aconselhável utilizar esta curva para a calibração.

Com o objetivo de avaliar o sistema PVGCV AAS frente à calibração com diferentes espécies de $\mathrm{Hg}$, foram construídas curvas analíticas com padrões de $\mathrm{Hg}^{2+}, \mathrm{CH}_{3} \mathrm{Hg}^{+}$e $\mathrm{C}_{2} \mathrm{H}_{5} \mathrm{Hg}^{+}$. Os valores de inclinação e coeficientes de correlação linear obtidos são apresentados na Tabela 3.

Tabela 3. Parâmetros de mérito para as curvas analíticas das diferentes espécies de $\mathrm{Hg}$

\begin{tabular}{ccc}
\hline & $\mathbf{a}\left(\mathbf{L}{\mathbf{~} \mathbf{g}^{-1}}^{-1}\right.$ & $\mathbf{R}$ \\
\hline $\mathbf{H g}^{2+}$ & 0,0120 & 0,9975 \\
$\mathrm{CH}_{3} \mathbf{H g}^{+}$ & 0,0138 & 0,9990 \\
$\mathrm{C}_{2} \mathbf{H}_{5} \mathbf{H g}^{+}$ & 0,0128 & 0,9922 \\
\hline
\end{tabular}

a: coeficiente angular

R: coeficiente de correlação da curva analítica

De acordo com os dados da Tabela 3, fica evidente a eficiência do sistema PVG-CV AAS em promover a redução para $\mathrm{Hg}^{0}$, tanto para as espécies organometálicas quanto para a forma inorgânica de $\mathrm{Hg}$. Os valores de inclinação demonstram que não houve diferença significativa nos valores de inclinação encontrados, o que reafirma a possiblidade de calibração apenas com padrões de $\mathrm{Hg}$ inorgânico, que se comparado com os padrões organometálicos, são mais baratos e estáveis.
De modo a avaliar a exatidão, foram realizados testes de adição e recuperação nas amostras de biodiesel com uma mistura das diferentes espécies de $\mathrm{Hg}$, conforme mencionado no item 2.5, na parte experimental, assegurando assim a capacidade do método desenvolvido para a determinação de $\mathrm{Hg}$ total, independente da espécie de $\mathrm{Hg}$ que possa estar presente ou não nas amostras de biodiesel. A Tabela 4 mostra os valores de concentração obtidos após as leituras em triplicata. 
Tabela 4. Resultados para os testes de adição e recuperação no biodiesel pela adição de misturas das diferentes espécies de $\mathrm{Hg}$ e calibração com padrão de $\mathrm{Hg}^{2+}$ utilizando o sistema PVG-CV AAS (média \pm desvio padrão, $n=3$ )

\begin{tabular}{|c|c|c|c|}
\hline $\begin{array}{c}\text { Valor adicionado } \\
\qquad\left(\mu \mathrm{g} \mathrm{L}^{-1}\right)\end{array}$ & $\begin{array}{c}\text { Valor encontrado } \\
\qquad\left(\mu g \mathrm{~L}^{-1}\right)\end{array}$ & DPR (\%) & $\begin{array}{c}\text { Recuperação } \\
\text { (\%) }\end{array}$ \\
\hline \multicolumn{4}{|c|}{ Biodiesel 1} \\
\hline 0 & $<\mathrm{LD}$ & & - \\
\hline 3,0 & $3,36 \pm 0,02$ & 0,6 & 111,9 \\
\hline 6,0 & $6,59 \pm 0,01$ & 0,1 & 109,8 \\
\hline 9,0 & $9,40 \pm 0,02$ & 0,2 & 104,4 \\
\hline \multicolumn{4}{|c|}{ Biodiesel 2} \\
\hline 0 & $<\mathrm{LD}$ & - & - \\
\hline 3,0 & $3,04 \pm 0,08$ & 2,6 & 101,3 \\
\hline 6,0 & $6,06 \pm 0,03$ & 0,5 & 101,0 \\
\hline 9,0 & $9,78 \pm 0,06$ & 0,6 & 108,6 \\
\hline \multicolumn{4}{|c|}{ Biodiesel 3} \\
\hline 0 & $<\mathrm{LD}$ & & - \\
\hline 3,0 & $3,04 \pm 0,04$ & 1,3 & 101,3 \\
\hline 6,0 & $6,09 \pm 0,06$ & 0,9 & 101,5 \\
\hline 9,0 & $9,60 \pm 0,09$ & 0,9 & 106,7 \\
\hline
\end{tabular}

DPR: desvio padrão relativo

Os valores de recuperação encontrados ficaram na faixa de 101,0 a $111,9 \%$ indicando a boa exatidão do método. A precisão foi confirmada a partir dos valores de desvio padrão relativo (DPR) que ficaram menores que $0,1 \%$. Estes resultados estão dentro dos valores de referência aceitáveis pela Association of Official Analytical Chemists (AOAC) ${ }^{20}$

Durante as análises não foi detectada nenhuma quantidade de $\mathrm{Hg}$ significativa nas três amostras de biodiesel investigadas, pois os valores obtidos ficaram abaixo do limite de detecção instrumental obtido, de acordo com os dados da Tabela 2. No entanto, foram encontrados trabalhos que realizaram a determinação de $\mathrm{Hg}$ em amostras de óleo diesel e de gasolina de postos em diferentes regiões do Brasil. Cassela e colaboradores ${ }^{23}$ encontraram concentrações de $\mathrm{Hg}$ em amostras de óleo diesel na faixa de 2,6 a 6,8 $\mu \mathrm{g} \mathrm{L}^{-1}$. Vale ressaltar que de acordo com as leis vigentes, todo 0 óleo diesel comercializado no território nacional deve possuir cerca de $10 \%$ de biodiesel em sua composição. Jesus e colaboradores ${ }^{24}$, Torres e colaboradores $^{25}$ e Brandão e colaboradores $^{26}$ encontraram concentrações de $\mathrm{Hg}$ em amostras de gasolina na faixa de 0,11 a $0,90 \mu \mathrm{g} \mathrm{L}^{-1}$.

Como citado anteriormente, existem na literatura diversas técnicas analíticas para determinação de $\mathrm{Hg}$ em diferentes tipos de amostras. Estas normalmente fazem uso da geração química de vapor convencional utilizando $\mathrm{SnCl}_{2}$ ou $\mathrm{NaBH}_{4}$ como agentes 
redutores. É nítido o interesse em aplicar estas técnicas para determinação elementar em amostras de biocombustíveis e combustíveis, devido à sensibilidade fornecida pelas mesmas e os bons valores de limite de detecção.

A Tabela 5 apresenta os limites de detecção e quantificação, as técnicas analíticas empregadas, $\mathrm{o}$ tratamento da amostra e o nível de concentração de $\mathrm{Hg}$ obtidos em diferentes amostras de combustíveis e biocombustíveis (etanol combustível, glicerina, gasolina, óleo diesel e biodiesel). Pode-se perceber que a técnica de geração fotoquímica de vapor acoplada à CV AAS que foi utilizada neste trabalho mostrouse sensível para a detecção de $\mathrm{Hg}$, apresentando resultados similares em relação aos valores de limites de detecção obtidos para as amostras de etanol combustível $^{7,12}$, glicerina ${ }^{13}$, gasolina ${ }^{24-26}$ e biodiesel $^{27}$ e até melhores que aqueles os obtidos para amostras de óleo diesel ${ }^{23}$. 0 preparo da amostra é relativamente simples, o que sugere sua viabilidade para a análise de amostras oleosas.

Tabela 5. Comparação de métodos para a determinação de Hg em diferentes amostras de combustível e biocombustível

\begin{tabular}{|c|c|c|c|c|c|c|c|}
\hline Amostra & $\begin{array}{c}\text { LD } \\
\left(\mu \mathrm{L}^{-1}\right)\end{array}$ & $\begin{array}{c}\text { LQ } \\
\left(\mu \mathrm{L}^{-1}\right)\end{array}$ & $\begin{array}{l}\text { DPR } \\
(\%)\end{array}$ & Técnica & Preparo da amostra & $\begin{array}{c}\text { Concentra } \\
\text { ção de } \mathrm{Hg} \\
\text { encontrad } \\
\mathrm{a}\left(\mu \mathrm{L} \mathrm{L}^{-1}\right)\end{array}$ & Referência \\
\hline *Biodiesel & 0,01 & 0,05 & $<0,1$ & PVG-CV AAS & $\begin{array}{c}2 \mathrm{~g} \text { de biodiesel }+11,8 \mathrm{~mL} \text { de } \mathrm{HCOOH}+ \\
\mathrm{CH}_{3} \mathrm{CH}_{2} \mathrm{OH}\end{array}$ & $<L D$ & - \\
\hline $\begin{array}{c}\text { Etanol } \\
\text { combustível }\end{array}$ & 0,05 & - & $<4,5$ & PVG-CV AAS & $\begin{array}{c}25 \mathrm{~mL} \text { de etanol combustível }+1,3 \mathrm{~mL} \\
\text { de } \mathrm{HCOOH} \text { ou } 2,5 \mathrm{~mL} \text { de } \mathrm{H}_{3} \mathrm{CCOOH}\end{array}$ & $<L D$ & [12] \\
\hline Biodiesel & 0,03 & - & $<8$ & $\begin{array}{l}\mathrm{FI}-\mathrm{CV} \text { AAS com } \\
\text { redutor } \mathrm{SnCl}_{2}\end{array}$ & $\begin{array}{c}1 \mathrm{~mL} \text { de biodiesel }+3,0 \mathrm{~mL} \text { de } \mathrm{HNO}_{3} \\
65 \% \text { (vqv) }+1,5 \mathrm{~mL} \text { Triton } \mathrm{X}-100\end{array}$ & $\begin{array}{c}\text { Biodiesel } \\
1: 2,2 \pm 0,2 \\
\text { Biodiesel } \\
2: 3,7 \pm 0,1\end{array}$ & [27] \\
\hline $\begin{array}{c}\text { Etanol } \\
\text { combustível }\end{array}$ & 0,05 & 0,17 & $<3,5$ & $\begin{array}{l}\mathrm{CV} \text { AAS com } \\
\text { redutor } \mathrm{NaBH}_{4}\end{array}$ & $\begin{array}{c}50 \%(\mathrm{v} / \mathrm{v}) \text { etanol combustível + } \\
50 \%(\mathrm{v} / \mathrm{v}) \text { água deionizada }\end{array}$ & $<L D$ & [7] \\
\hline Glicerina & 0,05 & 0,19 & $<6,7$ & PVG-CV AAS & $\begin{array}{c}5 \mathrm{~g} \text { de glicerina }+5,9 \mathrm{~mL} \text { de } \mathrm{HCOOH}+ \\
\qquad \mathrm{CH}_{3} \mathrm{CH}_{2} \mathrm{OH}\end{array}$ & $<L Q$ & [13] \\
\hline Gasolina & 0,1 & 0,3 & $<8$ & PVG-GF AAS & $66 \%$ de gasolina $+34 \%$ de propanol & $\begin{array}{c}\text { Gasolina 1: } \\
0,11 \\
\text { Gasolina 2: } \\
0,14 \\
\text { Gasolina 3: } \\
0,18\end{array}$ & [24] \\
\hline Gasolina & 0,08 & - & $<2,4$ & $\begin{array}{l}\text { CV-GF AAS com } \\
\text { coluna de } \mathrm{Au} \text { e } \\
\text { redutor } \mathrm{NaBH}_{4}\end{array}$ & $\begin{array}{c}25 \mathrm{~mL} \text { de gasolina }+25 \mathrm{~mL} \text { de } \\
\mathrm{CH}_{3} \mathrm{CH}_{2} \mathrm{OH}\end{array}$ & $\begin{array}{l}5 \text { tipos de } \\
\text { gasolina: } \\
0,40 \text { a } 0,90\end{array}$ & [25] \\
\hline Gasolina & 0,10 & - & $<6$ & $\begin{array}{l}\text { CV AAS com } \\
\text { redutor } \mathrm{NaBH}_{4}\end{array}$ & $\begin{array}{c}20 \mathrm{~mL} \text { de gasolina }+15 \mathrm{~mL} \text { de propanol } \\
+1 \mathrm{~mL} \text { de } \mathrm{HNO}_{3} 50 \%\end{array}$ & 0,11 a 0,27 & [26] \\
\hline Óleo diesel & 0,6 & 1,9 & $<11,7$ & $\begin{array}{l}\mathrm{CV} \text { AAS com } \\
\text { redutor } \mathrm{NaBH}_{4}\end{array}$ & $\begin{array}{c}20 \mathrm{~mL} \text { de óleo diesel }+2,5 \% \text { de Triton } \\
\quad \mathrm{X}-100+15 \%(\mathrm{v} / \mathrm{v}) \mathrm{HNO}_{3}\end{array}$ & $\begin{array}{l}\text { Diesel 1: } \\
2,6 \pm 0,3 \\
\text { Diesel 2: } \\
6,8 \pm 0,8\end{array}$ & [23] \\
\hline Biodiesel & 0,9 & - & $<5,4$ & GF AAS & $\begin{array}{l}0,5 \mathrm{~g} \text { de biodiesel }+0,25 \mathrm{~mL} \text { de } \mathrm{HNO}_{3}+ \\
\text { Triton } \mathrm{X}-100+\text { álcool isopropílico }\end{array}$ & $\begin{array}{c}\text { Biodiesel: } \\
23,2 \pm 0,2 \\
\mu \mathrm{g} \mathrm{kg}^{-1}\end{array}$ & [28] \\
\hline
\end{tabular}

DPR: Desvio padrão relativo

*Presente trabalho. 
PVG- CV AAS: Geração fotoquímica de vapor acoplada à Espectrometria de absorção atômica com vapor frio

FI-CV AAS: Espectrometria de absorção atômica com vapor e injeção em fluxo

CV AAS: Espectrometria de absorção atômica com vapor frio

PVG-GF AAS: Geração fotoquímica de vapor acoplada à Espectrometria de absorção atômica em forno de grafite

CV-GF AAS: Espectrometria de absorção atômica em forno de grafite com vapor frio

GF AAS: Espectrometria de absorção atômica em forno de grafite

\section{Conclusão}

O método desenvolvido usando a técnica de PVG-CV AAS apresentou adequada sensibilidade, com elevada precisão e exatidão, parâmetros estes atestados pelos excelentes valores de desvio-padrão e de recuperação obtidos, respectivamente.

A grande vantagem de se utilizar a geração fotoquímica de vapor para introdução da amostra na técnica de CV AAS está na simplificação do método, onde não é requerido um tratamento extensivo da amostra, apenas uma simples etapa de diluição em meio de ácido fórmico e álcool etílico. Os limites de detecção obtidos foram comparáveis àqueles dos métodos convencionais usados para determinar $\mathrm{Hg}$ por geração química de vapor em amostras como etanol combustível, glicerina, entre outras.

Embora as especificações atuais não contemplem a determinação e os níveis máximos permitidos para $\mathrm{Hg}$, o método desenvolvido é eficaz e viável para a análise de amostras oleosas como o biodiesel.

\section{Agradecimentos}

Os autores agradecem à Coordenação de Aperfeiçoamento de Pessoal de Nível Superior (CAPES) e a Fundação de Amparo à Pesquisa do Estado do Rio Grande do Sul (FAPERGS) pela concessão da bolsa de doutorado, ao Conselho Nacional de Desenvolvimento Científico e Tecnológico
(CNPq) pelo apoio financeiro através do projeto Universal 14/2014 (Processo 447552/2014-7) e pela bolsa de Produtividade em Pesquisa (Processo 310917/2013-1) e também a L'OREAL Brasil pelo auxílio financeiro através do Prêmio para Mulheres na Ciência de 2011.

\section{Referências Bibliográficas}

${ }^{1}$ Geris, R.; Santos, N. A. C.; Amaral, B. A.; Maia, I. S.; Castro, V. D.; Carvalho, J. R. M. Biodiesel de soja - reação de transesterificação para aulas práticas de química orgânica. Química Nova 2007, 30, 1369. [CrossRef]

${ }^{2}$ Lôbo, I. P.; Ferreira, S. L. C.; Cruz, R. S. Biodiesel: quality parameters and analytical methods. Química Nova 2009, 32, 1596. [CrossRef]

${ }^{3}$ Knothe, G.; Gerpen, J. V.; Krah, J.; Ramos, L. P. Manual de Biodiesel, São Paulo, 2006.

${ }^{4}$ Resolução ANP no 45 DE 2014. Disponível em:

<http://nxt.anp.gov.br/nxt/gateway.dll/leg/r esolucoes anp/2014/agosto/ranp\%2045\%20\%202014.xml>. Acesso em: 12 junho 2018.

${ }^{5}$ Vieira, M.A.; De Oliveira, L.C.C.; Gonçalves, R.A.; Souza, V. R.; Campos, R. C. Determination of As in Vegetable Oil and Biodiesel by Graphite Furnace Atomic Absorption Spectrometry. Energy \& Fuels 2009, 23, 5942. [CrossRef]

${ }^{6}$ De Oliveira, L. C. C.; Vieira, M. A.; Ribeiro, A. S.; Lisboa, M. T.; Gonçalves, R. A.; De Campos, R. C. Determination of Silicon in Vegetable Oil and Biodiesel by High- 
${ }^{7}$ Resolution Continuum Source Flame Atomic Absorption Spectrometry Using Sample Dilution with Xylene. Energy \& Fuels 2012, 26, 7041. [CrossRef]

${ }^{8}$ Almeida, I. L. S.; Coelho, N. M. M. Direct Determination of Inorganic Mercury in Ethanol Fuel by Cold Vapor Atomic Absorption Spectrometry. Energy \& Fuels 2012, 26, 6003. [CrossRef]

${ }^{9}$ Aranda, P. R.; Gásquez, J. A.; Olsina, R. A.; Martinez, R. A. G. Method development for $\mathrm{Cd}$ and $\mathrm{Hg}$ determination in biodiesel by electrothermal atomic absorption spectrometry with emulsion sample introduction. Talanta 2012, 101, 353. [CrossRef] [PubMed]

${ }^{10}$ Leopold, K.; Foulkes, M.; Worsfold, P. Methods for the determination and speciation of mercury in natural waters - A review. Analytica Chimica Acta 2010, 663, 127. [CrossRef] [PubMed]

${ }^{11}$ Vieira, M. A.; Ribeiro, A. S.; Curtius, A. J.; Sturgeon, R. E. Determination of total mercury and methylmercury in biological samples by photochemical vapor generation. Analytical and Bioanalytical Chemistry 2007, 388, 837. [CrossRef]

${ }^{12}$ Oreste, E. Q.; De Oliveira, R. M.; Ribeiro, A. S.; Silva, M. M. Determination of $\mathrm{Hg}$ in Biological Samples and Ethanol Fuel by Photochemical Vapor Generation after PreConcentration in a Gold Trap. Journal of the Brazilian Chemical Society 2017, 28, 1779. [CrossRef]

${ }^{13}$ Silva, C. S.; Oreste, E. Q.; Nunes, A. M. N.; Vieira, M. A.; Ribeiro, A. S. Determination of mercury in ethanol biofuel by photochemical vapor generation. Journal of Analytical Atomic Spectrometry 2012, 27, 689. [CrossRef]

${ }^{14}$ Lisboa, M. T.; Clasen, C. D.; Oreste, E. Q.; Ribeiro, A. S.; Vieira, M. A. Comparison between Vapor Generation Methods Coupled to Atomic Absorption Spectrometry for Determination of $\mathrm{Hg}$ in Glycerin Samples. Energy \& Fuels 2015, 29, 1635. [CrossRef]

${ }^{15}$ De Jesus, A.; Zmozinski, A. V.; Vieira, M. A.; Ribeiro, A. S.; Silva, M. M. Determination of mercury in naphtha and petroleum condensate by photochemical vapor generation atomic absorption spectrometry. Microchemical Journal 2013, 110, 227. [CrossRef]

${ }^{16}$ Wu, P.; He, L.; Zheng, C.; Hou, X.; Sturgeon, R. E. Applications of chemical vapor generation in non-tetrahydroborate media to analytical atomic spectrometry. Journal of Analytical Atomic Spectrometry 2010, 25, 1217. [CrossRef]

${ }^{17}$ He, Y.; Hou, X.; Zheng, C.; Sturgeon, R. E. Critical evaluation of the application of photochemical vapour generation in analytical atomic spectrometry. Analytical and Bioanalytical Chemistry 2007, 388, 769. [CrossRef] [PubMed]

${ }^{18}$ Bendicho, C.; Lavilla, I.; Pena-Pereira, F.; Romero, V. Green chemistry in analytical atomic spectrometry: a review. Journal of Analytical Atomic Spectrometry 2012, 27, 1831. [CrossRef]

${ }^{19}$ Sturgeon, R. E.; Luong, V. Photo- and thermo-chemical vapor generation of mercury. Journal of Analytical Atomic Spectrometry 2013, 28, 1610. [CrossRef]

${ }^{20}$ Instituto Nacional de Metrologia, Qualidade e Tecnologia (INMETRO). Orientação sobre validação de métodos analíticos. Documento de caráter orientativo, DOQ-CGCRE-08, 2010, 20p.

${ }^{21}$ Association of Official Analytical Chemists AOAC (US). AOAC Official methods of analysis. Appendix F: Guidelines for Standard Method Performance Requirements. Washington: AOAC, 2012.

${ }^{22}$ Golimowski, J.; Golimowska, K. UVphotooxidation as pretreatment step in inorganic analysis of environmental samples. Analytica Chimica Acta 1996, 325, 111. [CrossRef]

${ }^{23}$ Guo, X; Sturgeon, R. E; Mester, Z; Gardner, G. UV Vapor Generation for Determination of Selenium by Heated Quartz Tube Atomic Absorption Spectrometry. Journal of Analytical Chemistry 2003, 75, 2092. [CrossRef]

${ }^{24}$ Cassela, R. J.; Vicentino, P. O.; Brum, D. M. Development of a method for total $\mathrm{Hg}$ 
determination in oil samples by cold vapor atomic absorption spectrometry after its extraction induced by emulsion breaking. Talanta 2015, 132, 733. [CrossRef]

${ }^{25}$ De Jesus, A.; Sturgeon, R. E.; Liu, J.; Silva, $M$. M. Determination of mercury in gasoline by photochemical vapor generation coupled to graphite furnace atomic absorption spectrometry. Microchemical Journal 2014, 117, 100. [CrossRef]

${ }^{26}$ Torres, D. P.; Dittert, I. M.; Höhn, H.; Frescura, V. L. A.; Curtius, A. J. Microchemical Journal 2010, 96, 32. [CrossRef]

${ }^{27}$ Brandão G. P.; Campos, R. C.; Luna, A. S. Determination of mercury in gasoline by cold vapor atomic absorption spectrometry with direct reduction in microemulsion media.
Spectrochimica Acta Part B 2005, 60, 625. [CrossRef]

${ }^{28}$ Aranda, P. R.; Pacheco, P. H.; Olsina, R. A.; Martinez, L. D.; Gil, R. A. Total and inorganic mercury determination in biodiesel by emulsion sample introduction and FI-CV-AFS after multivariate optimization. Journal of Analytical Atomic Spectrometry 2009, 24, 1441. [CrossRef]

Aranda, P. R.; Gásquez, J. A.; Olsina, R. A.; Martinez, L. D.; Gil, R. A. Method development for $\mathrm{Cd}$ and $\mathrm{Hg}$ determination in biodiesel by electrothermal atomic absorption spectrometry with emulsion sample introduction. Talanta 2012, 101, 353. [CrossRef] 